\title{
Endovascular Thrombectomy in Acute-Onset Ischemic Stroke - beyond the Standard Time Windows: A Case Report and a Review of the Literature
}

\author{
Conan So ${ }^{a, b}$ Naveed Chaudhry ${ }^{b}$ Dheeraj Gandhic John W. Cole ${ }^{a, b, d}$ \\ Melissa Mottab \\ aUniversity of Maryland School of Medicine, Baltimore, MD, USA; ${ }^{b}$ Department of \\ Neurology, University of Maryland Medical Center, Baltimore, MD, USA; 'Department of \\ Radiology, University of Maryland Medical Center, Baltimore, MD, USA; dDepartment of \\ Neurology, Baltimore Veterans Affairs Medical Center, Baltimore, MD, USA
}

\section{Keywords}

Mechanical endovascular thrombectomy · Delayed thrombectomy $\cdot$ Ischemic stroke

\begin{abstract}
Endovascular thrombectomy following an acute ischemic stroke can lead to improved functional outcome when performed early. Current guidelines suggest treatment within $6 \mathrm{~h}$ after symptom onset. Recent studies including the DEFUSE-3 and DAWN trials demonstrate that some patients may benefit from thrombectomy up to 16 and $24 \mathrm{~h}$ after symptom onset, respectively. We present a case of delayed thrombectomy in a 43-year-old man with acute dysarthria, left-sided weakness, and visual neglect. Initial MRI/A demonstrated a small completed stroke and a thrombus in the right middle cerebral artery. Thirty-seven hours after symptom onset, his weakness acutely worsened. A repeat MRI revealed an unchanged core infarct
\end{abstract}




\section{Case Reports in Neurology}

volume and a cerebral angiogram suggested an abrupt occlusion of the right distal M1. Thrombectomy was performed with complete reperfusion and the patient's strength recovered following the procedure. We compared our clinical reasoning with the DEFUSE-3 and DAWN study criteria, and conclude that there is a subset of patients that may safely benefit from thrombectomy in later time windows beyond the trial criteria, especially in the setting of clinical examination of imaging mismatch.

(C) 2018 The Author(s)

Published by S. Karger AG, Basel

\section{Introduction}

Reperfusion is the cornerstone of acute ischemic stroke treatment. Early treatment with intravenous tissue plasminogen activator is associated with decreased mortality and better functional outcome [1]. Early endovascular thrombectomy also leads to improved outcomes for patients with proximal occlusions when started within $6 \mathrm{~h}$ of onset of symptoms [2]. The time windows for endovascular therapy have recently expanded driven by results from the DEFUSE-3 [3] and DAWN [4] trials, demonstrating that select patients can benefit from treatment beyond $6 \mathrm{~h}$. Using highly defined inclusion and exclusion criteria, these trials demonstrated favorable thrombectomy outcomes up to $16 \mathrm{~h}$ in DEFUSE-3 [3] and up to $24 \mathrm{~h}$ in DAWN [4]. The DAWN and DEFUSE-3 investigators included patients with ischemic strokes affecting the proximal middle cerebral artery (MCA) or intracranial internal carotid artery and stratified patients based on imaging and perfusion mismatch $[3,4]$. We present an ischemic stroke case of markedly delayed thrombectomy occurring $37 \mathrm{~h}$ after the patient was last known to be normal resulting in a good clinical outcome and contrast our clinical reasoning with the DAWN and DEFUSE-3 study criteria.

\section{Case Report}

A 43-year-old man developed acute-onset dysarthria, left-sided weakness, and visual neglect. The patient initially declined intravenous tissue plasminogen activator at an outside hospital due to his minor symptoms and was transferred to our Comprehensive Stroke Center with an NIH Stroke Score (NIHSS) [5] of 5 for further evaluation and treatment. MRI diffusionweighted imaging performed at $15 \mathrm{~h}$ after symptom onset demonstrated diffusion restriction in the right corona radiata with a small infarct volume of $5.4 \mathrm{~cm}^{2}$ (Fig. 1a). The susceptibilityweighted imaging sequences were compatible with a thrombus in the right MCA (Fig. 1b). The patient initially remained clinically stable; however, the patient's weakness worsened at $\sim 35 \mathrm{~h}$ after symptom onset with an increased NIHSS of 9. A repeat MRI revealed a right MCA territory ischemic infarction consistent in volume and distribution with the earlier study, with the susceptibility-weighted imaging again demonstrating a clot in the distal right MCA.

\section{Management}

Given the patient's declining clinical examination, with a stable relatively small diffusion deficit of $5.4 \mathrm{~cm}^{2}$, endovascular mechanical thrombectomy was offered. On cerebral angiogram, an abrupt occlusion of the right distal M1 was visualized (Fig. 1c). Two passes of a stent 


\section{Case Reports in Neurology}

retriever resulted in complete reperfusion to the right MCA (Fig. 1d), with a grade three thrombolysis in cerebral infarction score [6]. Reperfusion occurred $37 \mathrm{~h}$ after the patient was last known to be normal. The patient's strength improved following the procedure to an NIHSS of 7. He was discharged 3 days following thrombectomy to an acute rehabilitation hospital. He had a modified Rankin Scale (mRS) score of 1 at 6 months following thrombectomy with minimal residual deficits.

\section{Discussion}

While the upper time limit for endovascular thrombectomy of large anterior vessel occlusions in select cases has recently expanded up to $24 \mathrm{~h}$, the maximal treatment window remains unknown. A recent meta-analysis evaluated the benefit of thrombectomy in extended time windows after stroke symptom onset but featured few patients who underwent the procedure beyond $8 \mathrm{~h}$ [2]. The recent DEFUSE-3 and DAWN trials extended the role of delayed thrombectomy by selecting patients who presented between 6 and 16 or between 6 and $24 \mathrm{~h}$ after symptom onset, respectively $[3,4]$.

Our patient was somewhat similar to a DAWN trial patient. The DAWN investigators used diffusion-weighted imaging, FLAIR, and T2 MRI to calculate infarct volumes for MRI-based images, or noncontrast CT or perfusion CT for CT-based imaging. These images were then compared to the clinical NIHSS to identify those with "target mismatch" - a small core infarct volume but a large area of brain at risk for ischemia yet still potentially salvageable (Table 1). The study demonstrated a 2.1-point difference in the 90-day weighted mRS score in favor of the thrombectomy group, which translated into a $73 \%$ relative reduction of dependency in activities of daily living and a number needed to treat for any lower disability of 2.0 [4]. In addition, there was a $33 \%$ absolute increase in the number of patients achieving functional independence at 90 days (mRS score of $0-2$ ) when adjusted for baseline infarct volume, with a number needed to treat for this endpoint of 2.8 [4].

The DEFUSE-3 trial investigators likewise found significant clinical benefits for endovascular thrombectomy among patients with evidence of salvageable tissue at risk identified via imaging criteria (Table 1). Patients in the endovascular thrombectomy group plus standard medical therapy had an $28 \%$ absolute increase in the number of patients achieving functional independence at 90 days (mRS score of 0-2) compared to medical therapy only, with an adjusted odds ratio of 3.36 favoring thrombectomy when patients were stratified by age, core infarct volume, baseline NIHSS, and time from symptom onset to enrollment [3]. Given the positive findings from the DAWN and DEFUSE-3 trial, we compare our clinical reasoning for performing a delayed thrombectomy with the selection criteria of these two recent trials. Further, we demonstrated that the treatment window can be expanded and safely lengthened if similar selection criteria are utilized.

In our case, the patient underwent successful thrombectomy $37 \mathrm{~h}$ after symptoms onset. The location of occlusion and the image modality to assess tissue at risk/penumbra fit the selection criteria for both the DAWN and DEFUSE-3 trial. However, our patient had a lower NIHSS score than patients selected for the DAWN trial, with his initial NIHSS score of 5 that subsequently worsened to 9. In contrast, the minimum threshold NIHSS score in the DAWN trial patients under 80 was 10 . Although our patient's NIHSS remained below the trial criteria, 


\section{Case Reports in Neurology}

given his clinical worsening, but stable infarct volume in the setting of an established right MCA thrombus, the thrombectomy risk-benefit ratio appeared favorable. As such, we deemed that there was a strong possibility that salvageable tissue was present and proceeded with a delayed thrombectomy. The progression of deficits with unchanged infarct volume on repeat MRI was consistent with the clinical examination of imaging mismatch as sought in both the DEFUSE-3 and DAWN trials. The successful reperfusion salvaged the brain at risk.

While the ultimate goal of thrombectomy is to save penumbral brain tissue, the potential risk for symptomatic intracranial hemorrhagic conversion is always present. In the DEFUSE-3 and DAWN trials, the symptomatic intracranial hemorrhage (ICH) rates were 7 and $6 \%$, respectively. In our case, where a thrombectomy was performed beyond these time windows, the risk may be further elevated. Given these uncertainties, the authors suggest that highly select patients presenting more than $24 \mathrm{~h}$ after symptom onset could be considered for thrombectomy on a case-by-case basis. One might consider younger patients in particular, carefully weighing potential benefit against an uncertain, but elevated, ICH risk. Supporting this viewpoint, we note that despite the ICH risk, both the DAWN and DEFUSE-3 trials reported favorable outcomes. Ultimately, our case demonstrates that the relationships between the key parameters including clinical presentation (i.e., NIHSS), imaging mismatch, and the upper time limit for endovascular thrombectomy treatment remain uncertain. In such scenarios, it may be reasonable to consider a lower threshold to proceed with thrombectomy in younger patients who have an NIHSS lower than the selection criteria used in the trials (Table 1), especially if there is evidence of clinical examination of imaging mismatch. We suggest that future, prospective studies could potentially be designed to test this clinical scenario and treatment approach.

\section{Conclusion}

Endovascular thrombectomy is a proven treatment for patients with large proximal occlusions if performed within $6 \mathrm{~h}$ of stroke onset. Patients with clinical examination of imaging mismatch may respond especially well to delayed intra-arterial treatment, with recent trials supporting treatment up to $24 \mathrm{~h}$ after stroke onset, but this window may be potentially expanded further in highly select cases.

\section{Acknowledgements}

We thank José Merino, MD for his support and review of the manuscript. Dr. Cole's efforts on this project were supported in part by NIH grants U01 NS069208, R01 NS100178, and R01 NS105150; the US Department of Veterans Affairs, and the American Heart Association Cardiovascular Genome-Phenome Study (grant No. 15GPSPG23770000), and an American Heart Association Discovery Grant supported by Bayer Group (grant No. 17IBDG33700328). 


\section{Statement of Ethics}

The authors have no ethical conflicts to disclose.

\section{Disclosure Statement}

The authors declare that they have no potential conflicts of interest.

\section{Funding Sources}

This study did not receive any external funding.

\section{Author Contributions}

Conan So, MPH: Study conception and design; writing the manuscript; analysis and interpretation; critical revision of the manuscript; final approval.

Naveed Chaudhry, MD: Study conception and design; analysis and interpretation; critical revision of the manuscript; final approval.

Dheeraj Gandhi, MD: Analysis and interpretation; critical revision of the manuscript; final approval.

John W. Cole, MD, MS: Analysis and interpretation; critical revision of the manuscript; final approval.

Melissa Motta, MD, MPH: Analysis and Interpretation; critical revision of the manuscript; final approval; supervision and administrative support.

\section{References}

1 Emberson J, Lees KR, Lyden P, Blackwell L, Albers G, Bluhmki E, et al.; Stroke Thrombolysis Trialists' Collaborative Group. Effect of treatment delay, age, and stroke severity on the effects of intravenous thrombolysis with alteplase for acute ischaemic stroke: a meta-analysis of individual patient data from randomised trials. Lancet. 2014 Nov;384(9958):1929-35.

2 Saver JL, Goyal M, van der Lugt A, Menon BK, Majoie CB, Dippel DW, et al.; HERMES Collaborators. Time to Treatment With Endovascular Thrombectomy and Outcomes From Ischemic Stroke: A Meta-analysis. JAMA. 2016 Sep;316(12):1279-88.

3 Albers GW, Marks MP, Kemp S, Christensen S, Tsai JP, Ortega-Gutierrez S, et al.; DEFUSE 3 Investigators. Thrombectomy for Stroke at 6 to 16 Hours with Selection by Perfusion Imaging. N Engl J Med. 2018 Feb;378(8):708-18.

4 Nogueira RG, Jadhav AP, Haussen DC, Bonafe A, Budzik RF, Bhuva P, et al.; DAWN Trial Investigators. Thrombectomy 6 to 24 Hours after Stroke with a Mismatch between Deficit and Infarct. N Engl J Med. 2018 Jan;378(1):11-21.

5 Kwah LK, Diong J. National Institutes of Health Stroke Scale (NIHSS). J Physiother. 2014 Mar;60(1):61.

6 Higashida RT, Furlan AJ, Roberts H, Tomsick T, Connors B, Barr J, et al.; Technology Assessment Committee of the American Society of Interventional and Therapeutic Neuroradiology; Technology Assessment Committee of the Society of Interventional Radiology. Trial design and reporting standards for intra-arterial cerebral thrombolysis for acute ischemic stroke. Stroke. 2003 Aug;34(8):e109-37. 


\section{Case Reports in Neurology}
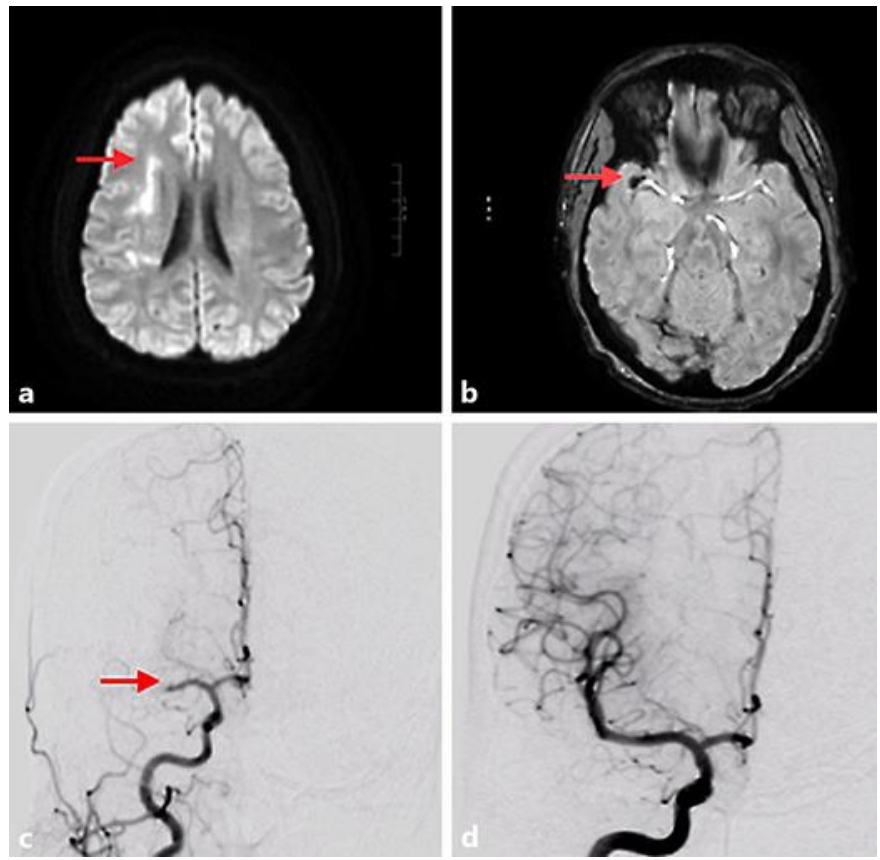

Fig. 1. a Magnetic resonance imaging of the axial brain with diffusion restriction (similar distribution on apparent diffusion coefficient imaging, not shown) involving the corona radiata (red arrow) consistent with ischemia. b Prominent blooming susceptibility artifact within the region of the right middle cerebral artery bifurcation compatible with thrombus (red arrow). c Right carotid injection on cerebral angiogram with abrupt occlusion of the M1 arterial segment of the right middle cerebral artery consistent with thromboembolism (red arrow). d Recanalization of the occluded vessel after mechanical thrombectomy. 
Table 1. Comparing DEFUSE-3, DAWN, and case report selection criteria

\begin{tabular}{|c|c|c|c|c|}
\hline Study & Lesion & $\begin{array}{l}\text { Time after } \\
\text { symptom } \\
\text { onset }\end{array}$ & Selection criteria & Infarct volume assessment \\
\hline DAWN & $\begin{array}{l}\text { Proximal MCA or } \\
\text { internal ICA }\end{array}$ & $6-24 \mathrm{~h}$ & $\begin{array}{l}\text { Group A: }>80 \text { years, NIHSS } 0-42 \text {, infarct } \\
<21 \mathrm{~mL} \\
\text { Group B: }<80 \text { years, NIHSS }>10 \text {, infarct }<31 \\
\text { mL } \\
\text { Group C: }<80 \text { years, NIHSS }>20 \text {, infract } 31- \\
51 \mathrm{~mL}\end{array}$ & $\begin{array}{l}\text { (1) Diffusion-weighted MRI at } \\
24 \text { h, followed by FLAIR or T2 } \\
\text { MRI } \\
\text { (2) Noncontrast CT or perfu- } \\
\text { sion CT }\end{array}$ \\
\hline DEFUSE-3 & $\begin{array}{l}\text { Proximal MCA or } \\
\text { internal ICA }\end{array}$ & $6-16 \mathrm{~h}$ & $\begin{array}{l}\text { Infarct }<70 \mathrm{~mL} \text {, ratio of volume of ischemic } \\
\text { tissue: infarct volume of } 1.8 \text {, penumbra } \\
\text { volume of }>15 \mathrm{~mL}\end{array}$ & $\begin{array}{l}\text { (1) Diffusion-weighted MRI } \\
\text { and MR perfusion scan } \\
\text { (2) Perfusion CT }\end{array}$ \\
\hline Case report & Proximal MCA & $37.5 \mathrm{~h}$ & $\begin{array}{l}\text { Worsening NIHSS score from } 5 \text { to } 9 \text { at } \\
\sim 35 \mathrm{~h} \text {, stable diffusion deficit with an in- } \\
\text { farct volume of } 5.4 \mathrm{~cm}^{2}\end{array}$ & $\begin{array}{l}\text { Diffusion-weighted MRI at } 15 \mathrm{~h} \\
\text { followed by susceptibility- } \\
\text { weighted MRI at } 34 \mathrm{~h}\end{array}$ \\
\hline
\end{tabular}

